\title{
Issues in the organisational and change context for innovations using ICT in higer education
}

\author{
Murray Saunders, Joël Bonamy and Bernadette Charlier \\ CSET (Centre for the Study of Education and Training,) Lancaster University, UK \\ GATE (Groupe d'Analyse et de Théorie Economique), Université Lumière Lyon, France \\ Facultés Universitaires N-D de la Paix, Cellule d'Ingénierie Pédagogique, Département \\ Education et Technologie, Namur, Belgium
}

\begin{abstract}
The RECRE@SUP project, under the European Union SOCRATESMINERVA initiative, was namely charged with investigating the context in which change related to the uses of ICT in teaching might take place within Higher Education (HE) institutional environments in context. This paper will outline an approach to this issue and provide an analytical framework grounded on the 20 cases of innovative teaching projects using ICT in the ten European University partners of the project. This framework, based on a qualitative analysis of the course leaders' experiences, helps to understand the change process and its contexts and to outline the main organisational issues associated with the introduction and dissemination for innovations using ICT in $\mathrm{HE}$.
\end{abstract}

Key words: innovation, higher education, organisational issues, ICT introduction

\section{INTRODUCTION AND THEORETICAL APPROACH}

To understand the change process related with uses of ICT in HE context, the RECRE@SUP project has chosen to describe and analyse 20 cases of course or curriculum. These cases, two per university, cover all the degrees of education from undergraduate to post-graduate level as well as a diversity of pedagogical approach and ICT uses (self-learning, collaborative projects, distance teaching). 
In terms of the design of the RECRE@SUP project and this paper, we consider changes which have incorporated use of ICT either in the justification for the course, in the curricular design or in its use. Unlike other research on the process of change and change in $\mathrm{HE}$ in particular, we have been interested in exploring the course leaders' conceptions of the change process, how they have 'visioned' change, and the organisational context for their course. Therefore the focus for this work has been on the way the process of change is conceptualised. In this analysis, we can see the way in which the courses are relatively 'loosely coupled' (see Weick, 1988) to the host organisation, providing the circumstance in which many conceptions of the organisational context for changes can occur, relatively 'free' from organisational determination. By 'coupling' we mean the extent to which parts of a system or sub-system are responsive to changes in other parts of the system. In a tightly coupled system for example, if intended changes at the level of policy were introduced from the centre, we would expect parts of the system to be responsive to these changes and changes might occur of the kind evoked by the policy. In a loosely coupled system, changes envisaged by the centre's policy are likely to produce far more varied responses from the sub-parts of the system or, indeed, no changes at all. In the HE cases we studied, it is clear that HE is loosely coupled in terms of curricular innovation based on ICTs. However, the project has been able to produce a typology of 'change conceptions' we think might be useful in understanding and planning innovation in this area. In order to develop these change strategies at the level of the course leader, we have developed and adapted a 'Theories of Change' approach to our work in analysing the organisational context of change in our case study courses. Essentially it adopts the perspective of evaluation and institutional research in which key participants are asked to consider how the activities they are undertaking will produce intended outcomes. In other words, what theory do the key stakeholders have (often implicit) about the way change can be produced by certain types of action? It might be a learning theory or it might be a theory about how organisations change (for example, pilots or experiments produce exemplars which are then adopted more widely in an organisation). The important thing is that key 'agents for change' are asked why they are doing what they are doing, and on what basis.

\section{METHODOLOGICAL FRAMEWORK}

The analysis reported here is based on 20 semi-structured interviews with the course leaders of 20 courses in our case study HE institutions. These interviews were analysed using a range of interrogatory concepts (the use of 
which is described below) by progressively focusing until we were working with a series of syntheses.

We built into our discussions a framework which we could use to make sense of the case study material we had synthesised. The framework we developed for discussing each case used a three dimensional structure. Each case was identified as one of the three categories of 'purpose' which will form a 'case type'.

- Developmental; concerned with course development i.e. course materials, teaching processes or learning processes in a discipline or subject area.

- Experimental; concerned with a fixed activity in time to test a way of using a teaching technology.

- Exploitational; concerned with the development of a course of study to exploit new customers or market.

We also used categories to denote how the case 'connects' to the wider organisational context in which it has been developed. These categories were derived from previous research in this area. We use 'military' metaphors to capture or conceptualise this process. A case might lie on a continuum between 'enclave' and 'bridgehead'.

A case might lie on a continuum between 'enclave' and 'bridgehead'. An enclave is a set of practices which exist in a larger organisational setting but which has characteristics which are distinctive; individuals within it subscribe self-consciously to a different culture (or way of doing things) to the organisational norm and there are clear organisational, temporal and sometimes spatial differences which distinguish it from its organisational setting.

As far as change is concerned, we are interested in the extent to which and under what conditions an enclave has an impact on the wider organisation. In other words it begins to challenge wider practices in the organisation and so transforming itself from an enclave to a 'bridgehead' or platform for wider developments. This is important because we are looking for theories or strategies for the way in which enclaves enable wider change to happen.

Other concepts which help to explain the relationship between an enclave and the wider context see enclaves as an 'emergent' culture within a 'dominant' culture in an organisation. We might say that as long as the new culture remains in an enclave then there is not necessarily any change at a wider organisational level. However, once an enclave begins to develop as a bridgehead, then it can emerge as an 'oppositional' culture to the dominant culture and challenge its dominant position in the organisation and there is a 'paradigm shift in practices'. 


\section{THEORIES OR IDEAS OF CHANGE}

\subsection{Practice-based exemplar}

There were examples where the interview discussed ways in which change might be produced by providing examples of how ICT might be used to improve the teaching and learning in a particular environment. The theory being that 'practical' embodiments of useful, interesting or innovative teaching will produce changes in individuals who are interested in adapting their practice or who feel change is inevitable or have other pressures to change. The idea of 'contagion' is interesting here. The theory of change embedded in this conception is relatively weak in that how and under what conditions an interesting exemplar would create changes within the wider system was not made explicit or remained opaque. This has been known as the epidemiological model of change in that a 'beacon' or enclave becomes a bridgehead by osmosis. Another take on this process may be that having a practice based exemplar is a necessary condition for change but is often not sufficient. Resources, and political will might also have to be in place. The great challenge without these other factors in place is how to move from an interesting but marginal enclave to an influential bridgehead.

\subsection{Resource driven/dependent}

This category of interviewees understands change in terms of the resourcing incentive. Change will occur when there is a financial or resource incentive, i.e. resources are offered on the basis of specified changes. In addition, change will not occur or at least is dependent on resources being made available to enable change to occur. This is an interesting example of 'categorically driven funding change'. Usually, and also in the examples we have in the project, this theory identifies the way a university centre forms a policy of development. It establishes criteria against which it invites bids for developmental funding. Only those bids which display the desired profile of characteristics receive funds. This model does imply relatively tight coupling between university departments and their institution.

\subsection{Institutional rhetorical support}

This category of interviewees has a theory of change which is based on the framework provided at the institutional level. It should involve resource 
allocations, and provides for legitimation for the changes which are sanctioned by institutional policy. That is to say change occurs when there is institutional power behind it. In most of the cases, institutional rhetorical support is not sufficient to produce changes. The process of change is interesting here in that many bottom-up exemplars run ahead of institutional rhetorical support. At some point though, institutional rhetorical support is required in order to promote wider adoption.

\subsection{Professional imperative (enriching student experience)}

This change theory is based on the idea of teaching professionalism. Because a 'good' teacher will be interested in providing up-to-date, interesting, well-supported material and processes which aid and deepen intellectual development, often collaboratively, he/she will be interested in using, experimenting and developing courses which take advantage of possibilities offered by ICT. Whereas resource dependency relies on the assumption of tight coupling between central resource allocators and those who will implement change 'on the ground' and is based upon the premise of the efficacy of sequential, hierarchical processes of 'top-down' change, other change theories stress the greater effectiveness of bottom-up approaches to the implementation of change. In particular the idea of communities of practice seems very evocative to us in that it focuses on the way individuals become socialised into academic cultures. According to critics of top-down change strategies (see for example Hjern and Hull, 1982), top-down models of change rely too heavily on the assumption of power and control exercised by those at the top of systems (such as central government policy-makers) or within organisations (such as university vicechancellors and senior management teams). In contrast, advocates of a bottom-up approach to change argue that it is vital to take into account the importance of such characteristics of loosely-coupled systems as: "power relations, conflicting interests and value systems between individuals and agencies responsible for making policy and those responsible for taking action" (Barrett and Fudge, 1981).

\subsection{Technological determinism}

This category of interviewees understands change in terms of the imperative embedded in ICT itself. There are three variations in this category. First, the technology itself requires students, teachers and 
managers and teaching arrangements to change practices because of its nature once introduced. Secondly, the institutions, agencies or centres have an imperative to have ICT at the core of their activities to gain commercial advantage, to gain access to the global 'learning market' and to deliver curricula and learning opportunities more effectively. Thirdly, there are changes in the expectations of the students in terms of learning support and ICT infrastructure. This imperative has produced or will produce changes in the institutions as they attempt to match these expectations. This imperative becomes more acute in the 'continuing education' environment. The theory here is that educational institutions are learner sensitive, particularly in a global market.

\section{VISIONARY CATEGORIES}

This category of outcome which was derived from interview provides a cross check on the change theories outlined above. Interestingly they do broadly validate the categories developed in the change theory analysis and refer to the visions of teaching and learning embodied in the course they were leading. However, they are not the same. They tend to focus on the pedagogic aims of the course itself. To that extent it is an account of the kind of teaching and learning the course leader considers desirable. The first category concerns a vision of teaching and learning enriched by the use of ICT. This was seen in three principle ways. First by increasing the participation of the students in deciding what they should learn and how. The second involves the student in receiving more, regular and qualitatively improved feedback, particularly on-line. The third involved course leaders in experiments with collaborative learning amongst learners, particularly on group projects. The second category of vision centred on using the course to experiment with ways of providing learning opportunities to dispersed groups, i.e. in distance learning mode. The third category was a vision in which the feasibility of using on-line resources was to be tested in environments in which there was institutional scepticism or collegiate doubt. The fourth category of vision was one in which the course would act as a vehicle by which institutional practice in teaching and learning might be changed to include a wider variety of teaching and learning method, while support might be incorporated, i.e. a cultural shift. This category of vision involved the repositioning of the institution in the global market to provide distant learning opportunities. 


\section{DISSEMINATION}

There were three broad views on dissemination derived from interviews which connect strongly with a 'theory of change':

- through academic channels. In this case dissemination was understood to take place through conventional academic routes via publication in text books or in journals.

- piloting for wider use. In this case dissemination was built into the genesis and rationale for the course in that through external pressures from the 'market', the institution had supported the case to test how to deliver learning in that mode. Other courses were already identified for a procedural 'roll out'. This was the only case where there was an explicit dissemination activity built into the original development.

- no dissemination strategy. In these cases, the question of dissemination had not arisen because the course leaders had not seen themselves as 'change agents' and their horizons were short. In effect, change was something that happened within not outside, their own professional practice.

- by demonstrating good practice. Interestingly, the cases which identified, demonstration or 'embodiments' of practice as a means of dissemination, did not in general have an idea of the way in which 'demonstrations' would be disseminated, i.e. under what circumstances enclaves of good practice become known by a wider institutional group, or become bridgeheads.

\section{OVERVIEW}

The categories used for both interrogatory concepts and analysis of the course leaders' discourses highlight how the change process is highly contextualised depending on complex and interlocking factors, such as the organisational structure (highly or loosely coupled) and strategies. Using this analytical framework, our further analysis will apprehend deeper the course leaders' change experience in context by describing change experiences, change strategies and configuration of course types which bring about change profiles. This analysis will be validated by the course leaders and confronted with the visions of other important actors of the innovation process (decision makers, colleagues, and technical as well as pedagogical agents). These representations of the organisational and change contexts for innovations using ICT in HE would provide 'bridging tools' to actors to live and manage this process in their own institution. 


\section{REFERENCES}

Barret, S. and Fudge, C. (eds.) (1981) Policy and action. London: Methuen

Hjern, B. and Hull, C. (1982) Implementation Research as Empirical Constitutionalism.

European Journal of Political Research, No. 2

Saunders, M. (1998) Organisational culture and the use of electronic support for occupational learning. Journal of Computer Assisted Learning, 14 , 2, 36-51

Saunders, M. (1998) Enclaves in British Schools: TVEI Implications. In J. Lauglo, and Lillis, S. (eds.) Vocationalising Education. Oxford: Pergamon

Saunders, M. (1986) The Innovation Enclave: Unintended Effects of TVEI Implementation.

TVEI Working Paper 1. CARE (University of East Anglia)

Weick, K. (1979) The Social Psychology of Organising. New York: Random House

Wenger, E. (1997). Communities of practice. Cambridge: Cambridge University Press

Yasha, M. and Gev (1995) Information Technology in Educational Management. London:

Chapman and Hall

Weick, K.E. (1976) Educational Organizations as Loosely Coupled Systems. Administrative

Science Quarterly, 21, 1-19

\section{BIOGRAPHIES}

Murray Saunders was founder of CSET (Centre for the Study of Education and Training, University of Lancaster, UK) and has worked as an evaluator for 20 years specialising in a wide range of areas. He is one of only a very small number of individuals in the UK with a personal chair specifically in evaluation and is immediate past president of the UK Evaluation Society (of which he was a founder member). Joël Bonamy is a researcher in the research centre Gate of the Centre National de la Recherche Scientifique at the University Lyon 2 . He is a specialist of the Service economy and analyses education and adult training as service activities for which information and communication technologies are crucial factors of change. He has participated to European projects (JITOL, Learnett, Recre@sup) with a view to evaluating organisational and institutional change linked with the use of ICT in education and vocational training. Bernadette Charlier is a teacher and researcher at the University of Namur (Belgium). She specialises in educational technology and adult education. She has co-ordinated EC projects (LEARN-NETT, RECRE@SUP) and collaborated in evaluation teams with $\mathrm{M}$. Saunders and J. Bonamy with a view to evaluating learning processes and products, training systems and the roles of the teachers. 